Editorial

\title{
Acknowledgement to Reviewers of Separations in 2019
}

Separations Editorial Office

MDPI, St. Alban-Anlage 66, 4052 Basel, Switzerland

Published: 20 January 2020

The editorial team greatly appreciates the reviewers who have dedicated their considerable time and expertise to the journal's rigorous editorial process over the past 12 months, regardless of whether the papers are finally published or not. In 2019, a total of 56 papers were published in the journal, with a median time to first decision of 17 days and a median time from submission to publication of 65.5 days. The editors would like to express their sincere gratitude to the following reviewers for their generous contribution in 2019:

\author{
Anticó, Enriqueta \\ Antolak, Hubert \\ Ashley, Jon \\ Avino, Pasquale \\ Baeza Baeza, Juan José \\ Barreca, Salvatore \\ Bartella, Lucia \\ Berthod, Alain \\ Berton, Paula \\ Biancolillo, Alessandra \\ Birkemeyer, Claudia \\ Boysen, Reinhard \\ Bridge, Candice \\ Bulejko, Pavel \\ Capuano, Andrea \\ Carasek, Eduardo \\ Carmona, Eric \\ Chien, Shih-Chang \\ Chisvert Sanía, Alberto \\ Ciulu, Marco \\ Cocovi Solberg, David Jaime \\ Corsaro, Carmelo \\ Costa, M. Clara \\ Danezis, George \\ Deineka, Victor \\ Devasurendra, Amila \\ Dimandja, Jean-Marie \\ Dotsikas, Yannis \\ Drabińska, Natalia \\ Dunphy, Michael J. \\ Dziurkowska, Ewelina \\ Ebersviller, Seth \\ Elliani, Rosangela
}

\author{
Fanali, Salvatore \\ Fidalgo, Alexandra \\ Fitzgerald, Neil \\ Flieger, Jolanta \\ Fogarassy, Csaba \\ Fontanals, Núria \\ Fretté, Xavier \\ Galarini, Roberta \\ German, Konstantin E. \\ Gonçalves, Virginia \\ Gunia-Krzyżak, Agnieszka \\ Harrison, Sabine \\ Heberger, Karoly \\ Held, Christoph \\ Herráez-Hernández, Rosa \\ Horváth, Krisztián \\ Houzé, Pascal \\ Hupp, Amber \\ Ito, Yoichiro \\ Jampilek, Josef \\ Jeleń, Henryk H. \\ Jirovetz, Leopold \\ Kabir, Abuzar \\ Kalogiannis, Stavros \\ Kaškonienè, Vilma \\ Keglevich, György \\ Kelley, Steven \\ Kertèsz, Szabolcs \\ Kim, Jaheon \\ Kim, Chuk Young \\ Kimbaris, Athanasios \\ Kirchhoff, Jon R. \\ Koel, Mihkel
}


Kosakowska, Olga

Kosyakov, Dmitry S.

Kragović, Milan

Kranjac, Marina

Lahtela, Ville

Le Ouay, Benjamin

Lee, Jaehwi

Liu, Zhiqian

Ljubenkov, Ivica

Lucena, Rafael

Lung, Ming-Yeou

Madej, Katarzyna

Majdan, Marek

Margui, Eva

Marta, Ferreiro-González

Mashima, Ryuichi

Medana, Claudio

Mesquita, Raquel B.R.

Miller-Schulze, Justin P.

Mitulovic, Goran

Moliner Martinez, Yolanda

Morini, Luca

Narduzzi, Luca

Nebot, Carolina

Neng, Nuno

Nikolakakis, Ioannis

Nischang, Ivo

Nunez, Oscar

Nycz, Jacek E

Oliveira, Hugo M.

Ovidi, Elisa

Paeng, Kijung

Paluch, Andrew S.

Pavic, Valentina

Pazdro, Ksenia

Pedzinski, Tomasz

Pereira, Vanda

Perugini, Paola

Peterson, Julia

Petrakis, Eleftherios A.

Peukert, Wolfgang.

Philippe, Allan

Picariello, Gianluca

Pimienta, Rodney Lacret

Pinto, Eugénia

Piotrowski, Paulina
Piriou, Yannick

Płotka-Wasylka, Justyna

Pohl, Pawel

Prima-García, Helena

Prosen, Helena

Rayson, Gary D.

Reyes-Garcés, Nathaly

Romero-González, Roberto

Rubino, Federico Maria

Russo, Marina

Rykowska, Iwona

Saiano, Filippo

Saielli, Giacomo

Samanidou, Victoria

Sander, Aleksandra

Seiner, Brienne N.

Şenilă, Marin

Sertić, Miranda

Shinomiya, Kazufusa

Siebenhofer, Matthäus

Skoczyńska, Ewa

Śliwa, Paweł

Snow, Nicholas

Sobisch, Titus

Socas-Rodríguez, Bárbara

Sovova, Helena

Sowa, Ireneusz

Stassen, Ivo

Sykora, David

Syrpas, Michail

Tanner, Eden

Torres-Padrón, M Esther

Trammell, Sam

Tranfo, Giovanna

Vehus, Tore Sandnes

Verdú-Andrés, Jorge

Videau, Patrick

Villaverde, Juan José

Vyviurska, Olga

Wahab, M. Farooq

Walter, Johannes

Włodarczyk, Maciej

Wuttke, Stefan

Yokota, Shinso

Zoltan-Istvan, Szabo

(C) 2020 by the author. Licensee MDPI, Basel, Switzerland. This article is an open access article distributed under the terms and conditions of the Creative Commons Attribution (CC BY) license (http://creativecommons.org/licenses/by/4.0/). 